\title{
SIMULATION OF A WHEELED VEHICLE DYNAMIC REGIMES IN LABORATORY CONDITIONS
}

The paper deals with simulation of wheeled vehicle dynamic regimes in laboratory conditions. Based on the analysis of static and dynamic characteristics of the vehicle and test equipment the calculation model was created that calculates the course of a loading moment on the simulator for the given vehicle and driving regime. The basic condition for the simulation is the requirement for reaching congruent courses of driving speeds at the same vehicle control both on the roadway and in the test room.

Key words: Driving simulator, simulation of loading, vehicle, test room, modelling

\section{Introduction}

When designing new solutions within research activities in the field of transportation technology it is impossible to avoid modelling of real systems either in the test room (physical model) or on the computer (mathematical model [1]). Such a modelling is implemented at creation of control algorithms and programmes for development of computer control systems. It is a significant change in designing the drives when model experimenting is used to a great extent. As early as at designing the vehicle drive there is an assumption that the chosen vehicle operational parameters are controlled from one centre. The cooperation is provided by control systems not only in crisis situations but also in usual operation. The decisive role in the control of drive operation, in cooperation of individual elements as well as the collaboration between the vehicle and driver is performed by automated systems. They are able to identify, process and optimize the parameters of vehicle running in the real time.

The vehicle is a multi-parametric system whose final behaviour depends not only on technical parameters but also on service conditions. Statistics show that human is the weakest link in transportation systems. Although intelligent assistant systems are frequently used in vehicles, human remains the least reliable and hardly predicted link in the transportation systems. The research in crisis situations in real operation is demanding (not only from the point of safety but also technically and economically), therefore, vehicle simulators are used for this purpose. The simulator allows driving a vehicle along a virtual track surrounded by the environment simulating the real conditions. The simulators consist of real parts of vehicle cockpits and surfaces providing the driver's view. Based on the driver's reactions in traffic situations, the simulator creates the conditions which are either similar or identical to the real vehicle behaviour.
A similar principle is applied in the simulators designed for examining changes in processes and energy transmission in the vehicle driving mechanism. The dynamic driving simulator is a new step in the development of virtual testing of drives. It provides researchers, designers and constructors with a deeper insight into the behaviour of a solved drive and offers a direct feedback to their activities from different points of view.

\section{Selected problems of drive modelling in a test room}

In simulation there is an effort to imitate a real system through a suitable model that is as similar as possible to the real equipment not only with its composition but also with its behaviour. It is suitable to set up the model in a test room from the real components of modelled equipment. Important part of such a model is the device for simulation of loading.

Basic characteristics are taken from steady-state regime measurements. Important requirements put on a loading device include ranges of parameters (moment, output, speed) and way of control. From the point of control, the requirement for the reproducibility of experiment is critical.

In non-steady state regimes it is necessary to take into account other parameters and properties characterizing dynamic properties. It concerns the tested drive and loading device. In mechanical systems it concerns, first of all, the moments of single elements inertia; in hydraulic and pneumatic systems it concerns the capacity resulting from elasticity of the used medium and its piping. An important factor of the model dynamics is its behaviour from the point of delays. It is necessary to consider the delay of processes themselves in the given modelled system and delays in control

\footnotetext{
* Robert Labuda, Andrej Kovalcik, Jan Repka, Vladimir Hlavna 
signals transmission. These dependences are gathered experimentally through measurements of transient characteristics.

\subsection{Influence of moment of inertia on dynamics of modelled system}

If the moment of inertia of the used brake is greater that the moment of inertia of the real system reduced to the drive of the tested drive (for example, of the combustion engine), the testing of dynamics of such a system is limited or completely deleted. A suitable solution in this case can be an inclusion of additional transfer into the tested drive and braking device. On the contrary, if the moment of inertia of the brake is smaller than the reduced moment of tested mechanism, the arrangement should provide the simulation of the additional loading. Doing so there are more options:

- insertion of additional transfer,

- insertion of additional mass (flywheel),

- process adaptation of the moment by means of a static component control.

If the mass of identical moment of inertia with the required moment (identical with the real drive value) is fixed to the shaft of the tested element, the control of loading device will be limited to the control of the static component of the moment only.

\subsection{Influence of delays in the controlled system and control mechanism}

The vehicle equipped with a combustion engine is a typical example of the system in which delays are demonstrated. The influence of delays is significant mainly because the real operation of such a system is in the majority of applications characterized by an unsteady-state regime. This is caused by an accidentally changing loading, changing interventions of service or automatic control.

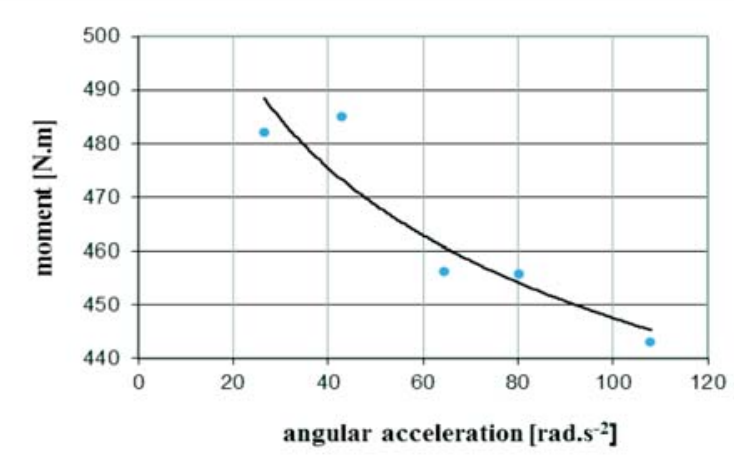

Fig. 1 Dependence of combustion engine moment on change speeds (at starting)

Demonstration of basic characteristics of engines as well as assessment of vehicle characteristics and their traction abilities (dynamic characteristics [2]) come out of static characteristics. Calculations of a vehicle dynamics are then influenced by certain inaccuracies. These can be found out experimentally, comparing static and dynamic courses. Fig. 1 shows the dependence of the achieved moment of the tested tractor engine $\mathrm{Z} 8604$ on the change speed. The change speed was simulated by a graded static loading at acceleration. The change in regime was induced by a jump change in fuel supply to the maximum value [3]. For calculation of vehicle dynamic characteristics [3] is, therefore, necessary to take into account the change of the combustion engine driving moment with change speed [4].

\section{Drive simulator}

\subsection{Description}

The drive simulator is a device that allows vehicle testing in laboratory conditions. The roadway (horizontal or sloping) is replaced by a roller of the test device. The analysis of force relations results from the contact of the vehicle and roadway. For the vehicle on a test track it is the roadway with given parameters, in a test room it is a roadway replaced by rollers of the test room.

\section{Steady-state regime in a roller test room.}

The objective is to express the volume of the brake moment on the test rollers that is needed for the equilibrium of forces $\left(F_{h}\right)$ between the vehicle wheels and test rollers. The driving force comes out of the equilibrium of moments on the driving wheel, Fig. 2a

$$
F_{h} \cdot r_{d}-M_{h}+M_{f}=0
$$

where

$$
F_{h}=\frac{M_{h}-M_{f}}{r_{d}}
$$

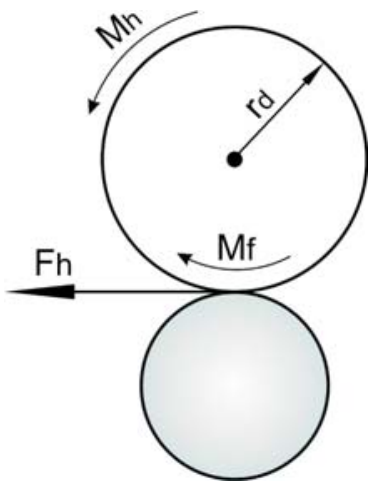

a)

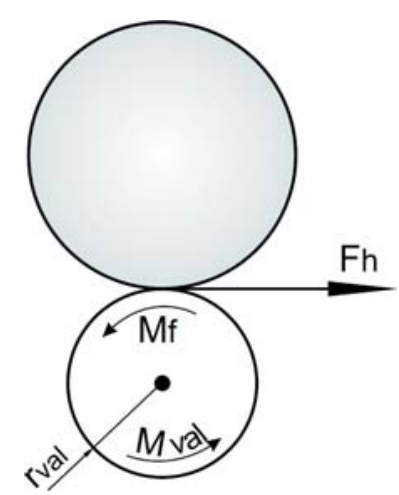

b)
Fig. 2 Scheme of force relations in the contact of vehicle and test rollers in a steady state

From the equilibrium of moments on the driven roller, Fig. $2 b$ 
$M_{v a l}+M_{f}=F_{h} \cdot r_{v a l}$

We can determine

$$
M_{\text {val }}=F_{h} \cdot r_{\text {val }}-M_{f}
$$

where

$F_{h} \quad$ - driving force on the vehicle wheels,

$M_{h}$ - driving moment on the vehicle wheels,

$M_{f}$ - moment of rolling resistance between vehicle wheels and rollers,

$r_{d}$ - dynamic radius of the driving wheel,

$M_{\text {val }}$ - moment on test rollers,

$r_{\text {val }}$ - test rollers radius.

From equations (2) and (4) the relation between moments on the driving wheel and braked rollers can be expressed

$$
M_{v a l}=M_{h} \cdot \frac{r_{v a l}}{r_{d}}-M_{f} \cdot\left(1+\frac{r_{v a l}}{r_{d}}\right)
$$

To achieve the values Mval on the rollers, the electrical dynamometer generating a particular brake moment is used.

\section{Dynamic - unsteady-state regime of the vehicle}

In an unsteady-state regime there are, apart from static load, resistances from inertia effects of moving and rotating masses of the tested vehicle, and inertia effects of the loading device. In loading simulation it is necessary to consider the fact that the tested vehicle stands still while the "roadway" - replaced by test rollers - is moving.

The volume of driving force on the vehicle wheels results from the motion equation [5]

$$
F_{h}=F_{v}+F_{f}+F_{s}+m_{v} \cdot \partial \cdot \frac{d v_{v}}{d t}
$$

For the equilibrium of moments on test rollers the following holds:

$$
F_{h} \cdot r_{v a l}=M_{v a l}+M_{f}+I_{v a l} \cdot \frac{d \omega_{v a l}}{d t}
$$

and the following driving force results:

$$
\begin{aligned}
& F_{h}=\frac{M_{v a l}+M_{f}}{r_{v a l}}+\frac{I_{v a l}}{r_{v a l}^{2}} \cdot \frac{d v_{v a l}}{d t} \\
& M_{b}^{d}=F_{\text {odp }} \cdot r_{d} \cdot i_{k_{-} d} \cdot \eta_{k_{-} d}+\frac{1}{r_{d} \cdot i_{k_{-} d}} \cdot\left(\begin{array}{l}
m_{v} \cdot r_{d}^{2} \cdot i_{k_{-} d}^{2} \cdot \eta_{k_{-} d}+4 \cdot I_{k} \cdot i_{k_{-} d}^{2} \cdot \eta_{k_{-d}}+ \\
+I_{s m} \cdot i_{s m_{-} d}^{2} \cdot \eta_{s m_{-d}}-4 \cdot I_{v a l} \cdot i_{v_{-} d}^{2} \cdot \frac{1}{\eta_{v_{-} d}}-I_{d}
\end{array}\right) \cdot \frac{d v_{v}}{d t}
\end{aligned}
$$

$I_{v a l}-$ moments of inertia of rotating rollers and parts among rollers and brake dynamometer reduced to the test rollers shaft, $\omega_{\text {val }}$ - roller angular velocity.

From the equality of the right sides of equations (6) and (8) the relation between the drive moment on rollers and resistances of the vehicle at slide less running $\left(v_{v}=v_{v a l}\right)$ can be expressed.

The simulation of vehicle loading is carried out through the control of the brake moment of rollers $M_{v a l}$ in compliance with the required acceleration of the vehicle $d v_{v} / d t$.

$$
\frac{M_{v a l}}{r_{v a l}}=F_{v}+F_{f}+F_{s}-\frac{M_{f}}{r_{v a l}}+\left(m_{v} \cdot \partial-\frac{I_{v a l}}{r_{v a l}^{2}}\right) \cdot \frac{d v_{v}}{d t}
$$

Equation (9) is the basis of the programme control of the moment on rollers according to the required acceleration. Modern drive simulators use the measurements of roller acceleration for control.

\section{Simulation of vehicle starting}

For loading simulation we chose the course of acceleration at the starting of Golf 1.4 TSI along the standardized test track with exactly defined parameters and respecting the conditions of official testing [6].

\subsection{Simulation of vehicle loading on the dynamometer shaft of a roller test room}

The loading simulation was performed on the dynamometer, point $D$ in Fig. 3. The simulation is influenced also by the increase in rolling resistance during the wheel rolling on test rollers opposed to rolling on the roadway $-M_{f} / r_{\text {val }}$. In an unsteady-state regime the control of brake moment will simulate the dynamic component representing inertia effects of moving masses of the vehicle and rotational masses of the drive. The control takes into account also inertia effects of rotating masses of loading device mechanisms (rollers, gear box, dynamometer rotor,...). The brake moment on the dynamometer shaft will be controlled in compliance with the where

$F_{v} \quad$ - vehicle air resistance,

$F_{f} \quad$ - rolling resistance between driving wheels and road,

$F_{s}$ - climbing resistance,

$m_{v}$ - vehicle total mass,

$\delta \quad$ - influence coefficient of rotating masses,

$v_{v}$ - circumferential speed on driving wheel (vehicle speed), where

$-i_{v d}=n_{v a l} / n_{d}$ transmission ratio between the dynamometer and test rollers,

$-i_{k d}=n_{k} / n_{d} \quad$ transmission ratio between the vehicle wheels and dynamometer shaft,

$-i_{s m d}=n_{s m} / n_{d}$ transmission ratio between the combustion engine and dynamometer, 


$-\eta_{k d}$
$-\eta_{v d}$
$-\eta_{s m d}$
$-F_{o d p}$
$-I_{k}, I_{s m}$

mechanical efficiency between the vehicle wheels and test room dynamometer,

mechanical efficiency between the rollers and test room dynamometer,

mechanical efficiency between the combustion engine and dynamometer,

drive resistance $\left(F_{v}+F_{f}+F_{s}\right)$,

moment of wheel inertia and combustion engine.
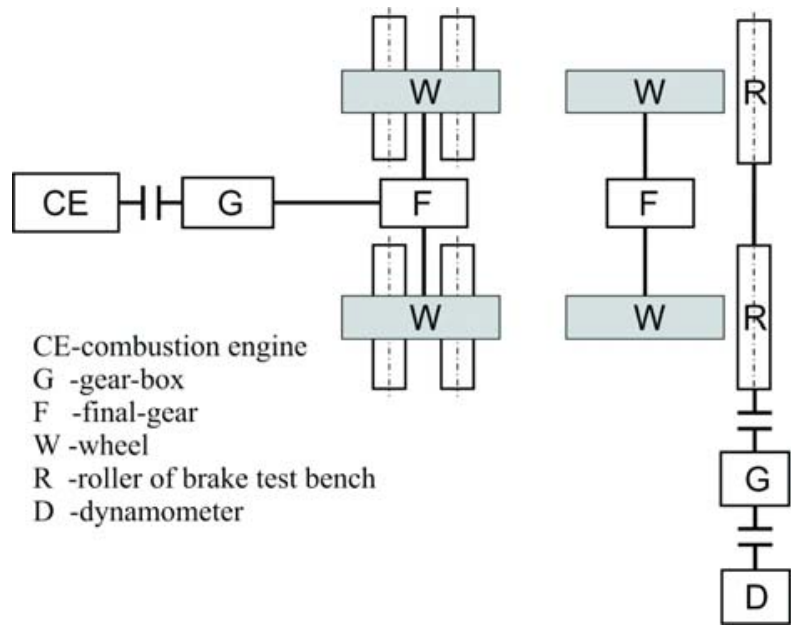

Fig. 3 Scheme of vehicle mechanism and output roller test room

\subsection{Simulation of vehicle loading on combustion engine shaft}

The system scheme in Fig. 4 shows a connection of the simulator directly to the combustion engine shaft. The simulator role is to induce the same loading on the combustion engine shaft as the loading from the transmission device and run on the real vehicle for the specified regime. Similarly as in equation (10) the relation for simulation of the moment on the combustion engine shaft is created:

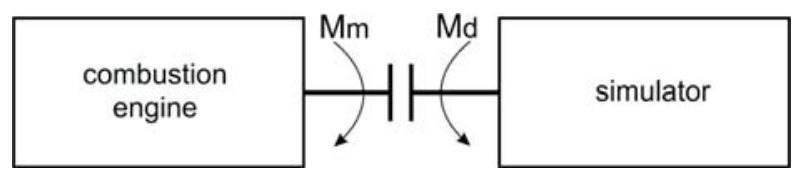

Fig. 4 Scheme simulator connection to combustion engine shaft where

$-i_{s m k}=n_{s m} / n_{k}$ transmission ratio between the combustion engine and vehicle wheels,

$-\eta_{s m k} \quad$ mechanical efficiency between combustion engine and vehicle driving wheels.

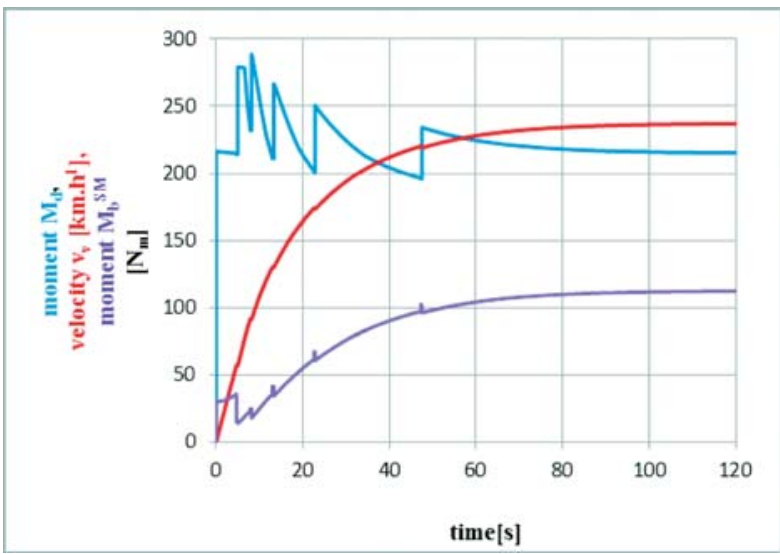

Fig. 5 Time characteristic of velocity for a vehicle run-up on dry asphalt; brake moments simulated on dynamometer shaft and on combustion engine shaft

\section{Results of simulation}

The basis for the simulation of loading is the time velocity characteristic achieved through the calculation in the program DYNAST [7]. To detune the programme of the brake moment simulation in the test room, the dynamic characteristic of a chosen vehicle starting was performed in the programme Dynast [7]. Fig. 5 illustrates the vehicle starting characterized by a time velocity characteristic (red line) and depiction of the courses of brake moments on the dynamometer shaft of the roller test room (blue line) and on the combustion engine shaft (violet line). Fig. 6 shows the relationship between the moment generated by the combustion engine and loading moment on the same shaft for the same time velocity characteristic (Fig. 5). The simulation of the brake moment on the combustion engine shaft for various roadway surfaces is illustrated in Fig. 7. In all the courses ( $M=$ konst.) the limitations are given by adhesion conditions. The curves Md follow the declining section of the outer speed characteristic of the combustion engine behind the point Mmax, (gear change at the revolutions of $4000 \mathrm{~min}^{-1}$ ).

$$
M_{b}^{s m}=F_{o d p} \cdot \frac{r_{d}}{i_{s m_{-} k} \cdot \eta_{s m_{-} k}}+\frac{i_{s m_{-} k}}{r_{d}} \cdot\left(m_{v} \cdot r_{d}^{2} \cdot \frac{1}{i_{s m_{-} k}^{2} \cdot \eta_{s m_{-} k}}+4 \cdot I_{k} \cdot \frac{1}{i_{s m_{-} k}^{2} \cdot \eta_{s m_{-} k}}+I_{s m}\right) \cdot \frac{d v}{d t}
$$




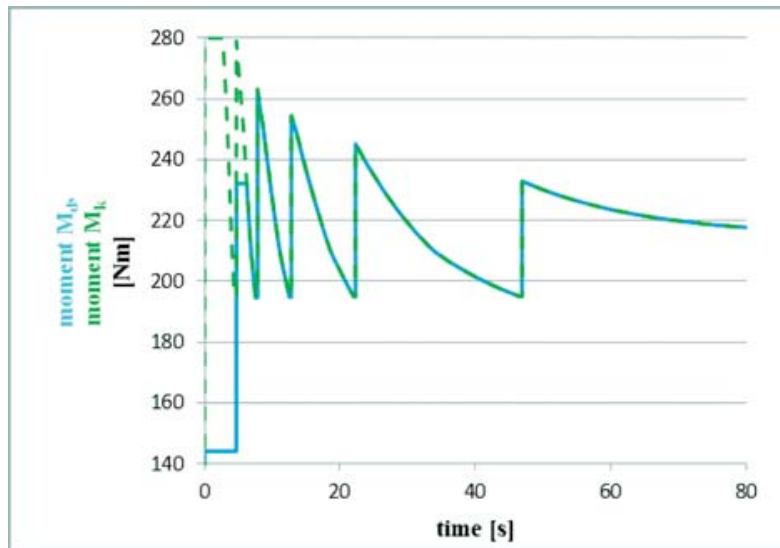

Fig. 6 Process of simulated brake moment on combustion engine shaft and moment generated by a combustion engine

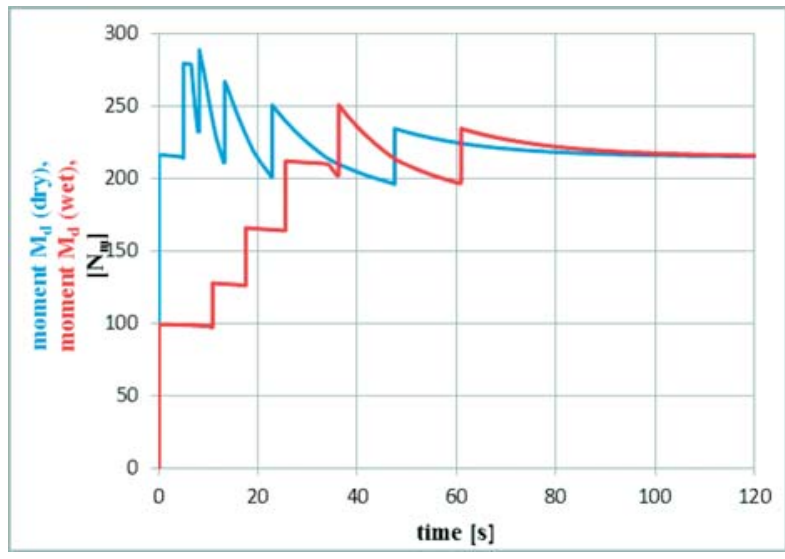

Fig. 7 Comparison of simulated brake moment on the engine shaft for dry and wet asphalt

\section{Acknowledgements}

This contribution was created within the framework of the project KEGA 038ZU-4/2011 and VEGA 1/0554/10, which are supported by the Ministry of Education of the Slovak Republic.

in the test room by which the real vehicle loading on the test bed is replaced. Another step of the solution will be the model of the whole vehicle system, of loading equipment and simulation of the vehicle dynamics at the programmed change in loading or in the engine driving moment.

\section{References}

[1] LAJCAKOVA, G., MELCER, J.: Dynamic Effect of Moving Vehicles on the Road Concrete Slabs, In: Communications - Scientific Letters of the University of Zilina, No. 3, 2011, ISSN 1335-4205.

[2] HLAVNA, V., GERLICI, J., LABUDA, R., LANG, A.: Means of Transport - Theory, EDIS - Publishing house of University of Zilina, 2006, ISBN 80-8070-498-8.

[3] LABUDA, R., ISTENÍK, R.: Results of Comparison Measurements Made on a Turbocharged Combustion Engine. In: Proc. of Transfer 2004, Section 6, Trencin, 2004, ISBN 80-8075-030-0

[4] SVOBODA, J.: The Theory of Means of Transport, Publishing house, CVUT in Prague, 2000,

[5] SEMETKO, J. et al.: Mobile Energy Means, Publishing Priroda, 1986

[6] VLK, F.: Testing and Diagnostics of Motor Vehicles - ${ }^{\text {st }}$ edition, Brno, 2001, p. 576, ISBN 80-238-6573-0.

[7] ISTENIK, R., FITZ, P.: Program System of Dynast, EDIS - Publishing house, University of Zilina, 2001. 